\title{
GLOBAL SUPERVENIENCE AND BELIEF
}

\begin{abstract}
Global supervenience of beliefs about physical states of affairs on such states has strongly counter-intuitive consequences about what beliefs we can nomologically hold. This is an argument against a global supervenience of all mental properties on physical ones, and, since that is implied by strong supervenience, also against that as the preferred materialist thesis.
\end{abstract}

\section{KAPLAN'S PROBLEM}

In (1983) David Kaplan has pointed out a restriction of possible worlds semantics. In the case of doxastic logic it has the consequence that not every possible proposition or state of affairs can be believed. Let $W$ be the set of worlds, to which an interpretation of a propositional language $\mathrm{L}$ of this logic refers. Sentences of the form $B(A)$ of $\mathrm{L}$ say that the person, whose beliefs are to be described in L - let's call him Max - , believes that the proposition expressed by $A$ obtains. If $S_{w}$ is the set of worlds, which for Max are in $w$ candidates for the real world, the sentence $B(A)$ is to be true in $w$ iff $S_{w}$ is a subset of the set $[A]$ of $A$-worlds. In the standard version of doxastic logic there are two conditions for the sets $S_{w}$ : (a) $S_{w}$ is a non-empty subset of $W$, the set of all possible worlds, and (b) If $w^{\prime}$ is in $S_{w}, S_{w^{\prime}}$ is identical with $S_{w}{ }^{1}$ If $W$ contains at least two elements, the function $S$ cannot be a mapping of $W$ onto the power set of $W$ minus the empty set, i.e. onto the set of all possible propositions. There are, then, possible propositions, $X$, for which there is no world $w$ such that $S_{w}=X$. Not every possible proposition can be believed by Max, either, since this would mean, that each proposition containing just one world could be believed. This would imply $\forall w \exists w^{\prime}\left(S_{w^{\prime}} \subseteq\{w\}\right)$, from which, with (a) and (b), we would obtain $S_{w^{\prime}}=S_{w}$ and therefore $\forall w\left(S_{w}=\{w\}\right)$. This would have the consequence that $B(A) \equiv A$ would be analytically true; being believed would coincide with being true. If

\footnotetext{
Journal of Philosophical Logic 23: 103-110, 1994.

(C) 1994 Kluwer Academic Publishers. Printed in the Netherlands.
} 
there are $n$ worlds in $W$, there are $2^{n}-1$ possible propositions. Each should be a possible doxastic attitude of Max, i.e. a possible $S_{w}$. For $n>1$ we have $2^{n}-1>n$, and for large $n$ the proportion $n / 2^{n}-1$ of the doxastic attitudes that may be realized in the worlds of $W$ to the possible ones becomes exceedingly small.

This doesn't mean that standard systems of doxastic logic are inadequate. For instance $B(B(A) \supset A)$ is a theorem of such logics - Max believes that his beliefs are correct. But it is possible that for some $A$ we have $B(A) \wedge \neg A$, i.e. that Max is mistaken. Therefore, if the operator $M$ stands for analytic possibility, i.e. truth in some world of the model, the principle

\section{P1*: $M(A) \supset M(B(A))-$ What is possible is possibly believed}

cannot be generally true. In particular it does not hold for some sentences $A$ expressing doxastic propositions. It should hold, however, for non-doxastic ones - we shall call them "objective" here - , for instance those that refer to the external, physical world. For sentences A expressing them it should even be logically possible that

$$
\text { P2*: } A \supset M(B(A) \wedge A)-\text { Each fact can be known. }
$$

Any logic is unacceptable in which this assumption implies omniscience. ${ }^{2}$

\section{O-INTERPRETATIONS}

We cannot simply distinguish sentences which express objective propositions syntactically, as these in which the belief operator $B$ does not occur, since sentential constants might have a doxastic meaning, too. A semantic distinction is indicated, therefore. As objective propositions are to constitute a separate segment of reality, we shall assume that the set $O$ of them is a complete Boolean algebra, i.e. it contains the complement of each element and the intersection of each of its subsets. We could define an equivalence relation $w \sim w^{\prime}$ between worlds by stipulating that it holds iff the same O-propositions obtain in both worlds. We shall, however, proceed the other way round and define $\mathrm{O}$ by an equivalence relation on $W$. $\mathrm{L}$ is now to contain an operator $O$, " $O(A)$ " standing for "It is an objective proposition that $A$ ". We also add the operator $N$ for 
analytic necessity to $L$. An appropriate concept of interpretations of L we call them $O$-models to distinguish them from standard ones - is this:

D1: An $O$-model of $\mathrm{L}$ is a quadruple $M=\langle W, \sim, S, V\rangle$ such that:

(1) $W$ is a non-empty set of worlds.

(2) $\sim$ is an equivalence relation on $W$.

(3) For all $w \in W$ :

(a) $\varnothing \neq S_{w} \subseteq W$,

(b) $w^{\prime} \in S_{w} \supset S_{w^{\prime}}=S_{w}$.

(4) For all $w \in W$ :

(a) $V_{w}(A) \in\{t, f\}$ and $w \sim w^{\prime}$ implies $V_{w}(A)=V_{w^{\prime}}(A)$ for all sentential constants $A$ and all $w^{\prime} \in W$.

(b) $V_{w}(\neg A)$ and $V_{w}(A \wedge B)$ are defined as usual.

(c) $V_{w}(B(A))=t$ iff $S_{w} \subseteq[A]$, where $[A]:=\left\{w^{\prime}: V_{w^{\prime}}(A)=t\right\}$.

(d) $V_{w}(N(A))=t$ iff $W \subseteq[A]$.

(e) $V_{w}(O(A))=t$ iff $\left.\forall w^{\prime} w^{\prime \prime}\left(w^{\prime} \sim w^{\prime \prime} \supset V_{w^{\prime}}(A)=V_{w^{\prime \prime}}(A)\right)\right)$.

All atomic sentences, then, are to express objective propositions, and a proposition is objective if it does not distinguish between equivalent worlds. $\mathrm{O}$, the set of objective propositions, is the set $\left\{X \subseteq W: \forall w w^{\prime}\right.$ $\left.\left(w \sim w^{\prime} \wedge w \in X \supset w^{\prime} \in X\right)\right\}$, so that we have $w \sim w^{\prime}$ iff $\forall X(X \in O \supset$ $\left.\left(w \in X \equiv w^{\prime} \in X\right)\right)$.

The ideas behind $\mathbf{P 1}^{\star}$ and $\mathbf{P 2}^{\star}$ can now be expressed by

P1: $O(A) \wedge M(A) \supset M(B(A))-$ Objective propositions can be believed

P2: $O(A) \wedge A \supset M(B(A) \wedge A)-$ Objective facts can be known.

In these versions the postulates do not have the unacceptable consequences of $\mathbf{P 1}^{*}$ and $\mathbf{P 2}^{*}$ anymore.

The set of sentences true in all $O$-models remains the same, if we add to D1 condition:

$$
w \sim w^{\prime} \wedge S_{w}=S_{w^{\prime}} \supset w=w^{\prime} .
$$

Logical truth, therefore, is not affected by identifying worlds that are objectively equivalent and in which Max holds the same beliefs.

A sentence of $\mathrm{L}$ not containing the operator $O$ is true in all standard 
models iff it is true in all $O$-models. If standard models are extended to sentences of the form $O(A)$ in such a way that for all sentences $C$ $N(O(C))$ holds, a sentence with $O$-occurrences is true in all standard models if the sentence $A^{\prime}$ obtained from $A$ by replacing $O$ everywhere by $O O$ is true in all $O$-models. For in these models $N(O(O(C)))$ is true for all $C$.

Regarding the connexions between objective and doxastic propositions we can distinguish two extreme cases of $O$-models $M=\langle W, \sim, S, V\rangle$ :

D2: $M$ is maximal iff $\forall w w^{\prime}\left(w \sim w^{\prime} \supset S_{w}=S_{w^{\prime}}\right) . M$ is minimal iff $\forall w X\left(X \in O \wedge X \neq \varnothing \supset \exists w^{\prime}\left(w^{\prime} \sim w \wedge S_{w^{\prime}} \subseteq X\right)\right)$.

For maximal $O$-models we have $w \sim w^{\prime} \supset w=w^{\prime}$, using (3c). Therefore all propositions are objective, and maximal $O$-models coincide with standard ones. Minimal $O$-models, on the other hand, are such that for every world $w$ and every possible objective proposition $X$ there is a world $w^{\prime}$, equivalent to $w$, in which Max believes that $X$ obtains; every such proposition can be believed under all possible objective conditions. Let $\llbracket w \rrbracket$ be the equivalence class for $w$, i.e. the set $\left\{w^{\prime}: w \sim w^{\prime}\right\}$. Then minimal $O$-models are such that $\forall w w^{\prime} \exists w^{\prime \prime}\left(S_{w^{\prime \prime}} \subseteq\left[w^{\prime}\right] \wedge w^{\prime \prime} \sim w\right)$. The corresponding postulate in $\mathrm{L}$ is

$$
\text { P3: } O(A) \wedge O(C) \wedge M(A) \wedge M(C) \supset M(B(A) \wedge C) \text {. }
$$

It implies the analytical independence of beliefs about objective propositions from the objective propositions themselves, and therefore a minimal correlation between them. Every consistent set of such beliefs is compatible with every consistent set of objective propositions. This is a realistic conception of the objective states of affairs, as a domain that is independent from our beliefs about it. That we hold certain beliefs about the physical world does not logically imply anything about its actual condition; our assumptions about it may in principle always turn out to be mistaken. This independence may be expressed by

$$
\text { P3a: } O(A) \wedge M(B(A)) \wedge O(C) \wedge M(C) \supset M(B(A) \wedge C) \text {. }
$$

Since $M(B(A))$ implies $M(A)$ this is a consequence of $\mathbf{P 3}$. P3 is stronger than P3a in so far as $M(A) \wedge O(A)$ does not imply $M(B(A))$. 
There are $O$-models in which $\mathbf{P 3}$ holds. Let $W_{0}$ be some non-empty set of worlds, $W_{1}$ the set of non-empty subsets of $W_{0}$, and $W=W_{0} \times W_{1}$, i.e. the set of pairs $\langle w, Z\rangle . w, w^{\prime}, \ldots$ are to be elements of $W_{0}, Z, Z^{\prime}, \ldots$ those of $W_{1}$, and $v, v^{\prime}, \ldots$ elements of $W$. Let $S_{\langle w, z\rangle}$ be $Z \times\{Z\}$, and $\langle w, Z\rangle \sim\left\langle w^{\prime}, Z^{\prime}\right\rangle$ iff $w=w^{\prime}$. Then the sets $S_{v}$ satisfy the conditions from D1, 3. We have $X \in O \equiv \exists Z\left(Z \subseteq W_{0} \wedge X=Z \times W_{1}\right)$, and $\forall v v^{\prime} \exists v^{\prime \prime}\left(v^{\prime \prime}\right.$ $\left.\sim v \wedge S_{v^{\prime \prime}} \subseteq\left[v^{\prime}\right]\right)$. For if $v=\langle w, Z\rangle$ and $v^{\prime}=\left\langle w^{\prime}, Z^{\prime}\right\rangle$, we may set $v^{\prime \prime}=$ $\left\langle w,\left\{w^{\prime}\right\}\right\rangle$. Then $v^{\prime \prime} \sim v$ and $S_{v^{\prime \prime}}=\left\{w^{\prime}\right\} x\left\{\left\{w^{\prime}\right\}\right\} \subseteq\left[v^{\prime}\right]=\left\{w^{\prime}\right\} \times W_{1}$.

\section{GLOBAL SUPERVENIENCE}

In discussions of global supervenience (GS, for short) it is mostly assumed that physical propositions form a complete Boolean algebra. ${ }^{3}$ If we define our equivalence relation $w \sim w^{\prime}$ in such a way that it holds iff the same physical propositions obtain in $w$ and $w^{\prime}, \mathrm{O}$ is the set of these propositions. If $X$, again, is a subset of $W,\left\{w: S_{w} \subseteq X\right\}$ is the doxastic proposition, that Max believes $X$ to obtain. In $w$ and $w^{\prime}$ the same doxastic propositions hold if $S_{w}=S_{w^{\prime}}$. An analytic GS of doxastic states of affairs on physical ones would mean: If two worlds differ as to the truth of some doxastic propositions, they also differ as to the truth of some physical propositions. This can be expressed by

$$
\forall w w^{\prime}\left(w \sim w^{\prime} \supset S_{w}=S_{w^{\prime}}\right)
$$

If that holds, we have a maximal $O$-model, each proposition is objective, and postulates $\mathbf{P 1}$ and $\mathbf{P 2}$ coincide with $\mathbf{P 1}^{\star}$ and $\mathbf{P 2}^{\star}$. But then we are faced again with the difficulties from which we started in Section I. According to (1) each physical proposition can be known only if for all sentence $A$ we have $B(A) \equiv A$, for instance. In each world in which Max is not omniscient, there are then physical facts that he cannot know for analytical reasons, and not just a few, but the overwhelming mass of them, as we have seen. According to (1), furthermore, every doxastic proposition is identical with a physical one, ${ }^{4}$ so that there can be no independence of physical facts from doxastic ones. (1), therefore, is not compatible with a realistic conception of physical reality.

Those are all strongly counter-intuitive consequences of an analytical GS in the form of (1). Generally, however, GS is not defined as an analytical, but as a nomological relation. Let $r$ be a relation of 
accessibility such that $w r w^{\prime}$ iff the same laws of nature hold in $w$ and $w^{\prime}$. $r$, then, is an equivalence relation. Let $r_{w}=\left\{w^{\prime}: w r w^{\prime}\right\}$ be the set of the worlds in which the same natural laws hold as in $w$, and let $w_{0}$ be the real world. For $r_{w_{0}}$ we also write $r_{0} . N_{0}$ is to be an operator for nomologic necessity with the truth condition: $V_{w}\left(N_{0}(A)\right)=t$ iff $r_{w} \subseteq[A] . M_{0}$ is to express the corresponding nomological possibility.

A nomological GS of doxastic on physical states of affairs holds, if we have

$$
\forall w w^{\prime}\left(w, w^{\prime} \in r_{0} \wedge w \sim w^{\prime} \supset S_{w}=S_{w^{\prime}}\right) .
$$

Materialists not only assume a GS of doxastic, but of all states of affairs on physical ones. For them, then, natural laws are also physical propositions. Since $\mathrm{O}$ is a complete Boolean algebra, this also holds for the conjunction of all natural laws, holding in a world. Then $r_{w}$ is in $\mathrm{O}$, and we obtain

$$
\forall w w^{\prime} w^{\prime \prime}\left(w \sim w^{\prime} \supset\left(w \in r_{w^{\prime \prime}} \equiv w^{\prime} \in r_{w^{\prime \prime}}\right)\right) .
$$

Because of the symmetry of $r$ this is equivalent with

$$
\begin{aligned}
& \forall w w^{\prime}\left(w \sim w^{\prime} \supset r_{w}=r_{w^{\prime}}\right)-\text { In equivalent worlds the same } \\
& \text { laws of nature hold. }
\end{aligned}
$$

Now, weakening (1) to (2) does not prevent Gs from having counterintuitive consequences. First, assume $O(A)$ and let $A^{\prime}$ express the proposition $\left\{w: w \in r_{0} \wedge S_{w} \subseteq[A]\right\}$. Then we have (i) $O\left(A^{\prime}\right)$, (ii) $N_{0}(B(A) \equiv$ $\left.A^{\prime}\right)$ and (iii) $N\left(B\left(A^{\prime}\right) \supset B(A)\right)$. (i) follows from (2) and (3). In the case of (iii), if we have $S_{w} \subseteq\left[A^{\prime}\right]$, there is a world $w^{\prime}$ such that $w^{\prime} \in S_{w}$ and therefore $S_{w^{\prime}} \subseteq X$; since $S_{w}=S_{w^{\prime}}$ we then also have $S_{w} \subseteq X$. Because of $O(A)$ and (i) we also have $O\left(A^{\prime} \wedge \neg A\right)$, and from that we obtain $M\left(A^{\prime} \wedge \neg A\right) \supset M\left(B\left(A^{\prime} \wedge \neg A\right)\right)$ by P2. From $M\left(A^{\prime} \wedge \neg A\right)$, therefore, we would obtain $M\left(B\left(A^{\prime}\right) \wedge B(\neg A)\right)$ and with (iii) $M(B(A) \wedge B(\neg A))$. Hence $M\left(A^{\prime} \wedge \neg A\right)$ cannot be true, i.e. we have $N\left(A^{\prime} \supset A\right)$ and in view of (ii) $N_{0}(B(A) \supset A)$. From the assumption that Max can believe every possible objective proposition, we thus get the absurd conclusion by (2) that his beliefs are nomologically true.

Furthermore, although the realistic independence postulate P3a does not exclude nomological correlations between doxastic and physical states of affairs, together with (2) it leads to quite implausible 
assumptions about analytical restrictions as to what can be believed. First, any physical proposition which may analytically be believed is believed with nomological necessity. That is

$$
O(A) \wedge M(B(A)) \supset N_{0}(B(A)) \text {. }
$$

Semantically P3a corresponds to the assumption $\forall X Y\left(X \in \mathrm{O} \wedge \exists w\left(S_{w}\right.\right.$ $\left.\subseteq X) \wedge Y \in O \wedge Y \neq \varnothing \supset \exists w^{\prime}\left(S_{w^{\prime}} \subseteq X \wedge w^{\prime} \in Y\right)\right)$. This implies $\forall X w^{\prime \prime}$ $\left(X \in O \wedge \exists w\left(S_{w} \subseteq X\right) \wedge w^{\prime \prime} \in r_{0} \supset \exists w^{\prime}\left(w^{\prime} \in r_{0} \wedge S_{w^{\prime}} \subseteq X \wedge w^{\prime} \sim w^{\prime \prime}\right)\right)$, since $w^{\prime \prime} \in r_{0}$ and $w^{\prime} \sim w^{\prime \prime}$ imply $w^{\prime} \in r_{0}$ according to (3). But from $w^{\prime}$, $w^{\prime \prime} \in r_{0}$ and $w^{\prime \prime} \sim w^{\prime}$ we obtain $S_{w^{\prime \prime}}=S_{w^{\prime}}$ by (2). Therefore we have $\forall X$ $\left(X \in O \wedge \exists w\left(S_{w} \subseteq X\right) \supset \forall w^{\prime \prime}\left(w^{\prime \prime} \in r_{0} \supset S_{w^{\prime \prime}} \subseteq X\right)\right)$, and that is the semantic correlate to (4).

Secondly, what is believed about the physical world in two analytically possible worlds would always have to be compatible, i.e.

$$
O(A) \wedge O(C) \wedge M(B(A)) \wedge M(B(C)) \supset M(A \wedge C)
$$

From the antecedent of (5) we obtain $N_{0}(B(A \wedge C))$ by (4), and, with $M(B(A)) \supset M(A)$ for all $A, M(A \wedge C)$. Finally, since $O(A)$ implies $O(\neg A)$,

$$
O(A) \wedge M(B(A)) \supset \neg M(B(\neg A))
$$

is a consequence of (5): For no physical proposition it is both analytically possible to believe it to be true and analytically possible to believe it to be false. This is not only implausible, but absurd. But then GS in the sense of $(2)$ is incompatible with a realistic conception of physical reality in the sense of P3a.

In our discussion we have used a rather narrow concept of belief. We have only considered a propositional language and only beliefs of one person; we have just referred to beliefs at a certain moment and have employed a strictly rational concept of belief, not fitted for the description of factual systems of assumptions, since they are not closed with respect to logical consequences. A generalization for languages of predicate logic and the beliefs of several persons, however, would not make any essential difference. More important would be an account of doxastic and physical changes in the different worlds, because we could then formulate intuitive assumptions about what we may come to believe more adequately, but we should still arrive at the same consequences. 
We still have no satisfactory descriptive concept of belief, but if beliefs do not refer to propositions as sets of worlds, but rather to entities composed of objects and their attributes, the number of possible states of belief surpasses that of possible worlds even farther, so that our argument would only be strengthened.

\section{NOTES}

1 For doxastic logic, cf. for instance Lenzen (1980).

${ }^{2}$ Knowledge, normally, is not defined as true conviction, but as true and well-founded conviction. This, however, is not an important point in our context. Well-founded beliefs are not truer than true beliefs, and they have no higher subjective probability either. They, too, can be wrong. Well-founded beliefs are best conceived of as beliefs that have been formed according to intersubjective standards of rationality, cf. Legris (1990).

3 The notion of GS for properties has been introduced by Hellman and Thompson in (1975), for propositions by Haugeland in (1982). Cf. also Kim (1984) and Kutschera (1992).

${ }^{4}$ Let $Y=\left\{w: S_{w} \subseteq Z\right\}$ be a doxastic proposition. For $X=\cup\{[w]: w \in Y\}$, we have $X \in O$ and $X=Y: w \in Y$ implies $[w] \subseteq X$, i.e. $w \in X$, and for $w \in X$ we have $[w] \subseteq X$, hence there is a $w^{\prime} \in Y$ such that $w^{\prime} \sim w$. By (1) we obtain $S_{w}=S_{w^{\prime}}$, and therefore $w \in Y$. Cf. Bacon (1986).

\section{REFERENCES}

Bacon, J. Supervenience, necessary coextension, and reducibility, Philosophical Studies 49 (1986), 163-76.

Haugeland, J. Weak supervenience, American Philosophical Quarterly 19 (1982), 93-103.

Hellman, G. and Thompson, F. Physicalism: Ontology, determination, and reduction, Journal of Philosophy 72 (1975), 551-64.

Kaplan, D. A problem in possible world semantics, Abstracts of the 7th International Congress of Logic, Methodology and Philosophy of Science, Salzburg, vol. 2 (1983), 8385.

Kim, J. Concepts of supervenience, Philosophy and Phenomenological Research 65 (1984), 257-70.

Kutschera, F. V. Supervenience and reductionism, Erkenntnis 36 (1992), 333-343.

Legris, X. Eine epistemische Interpretation der intuitionistischen Logik, Würzburg

(Königshausen und Neumann) 1990.

Lenzen, W. Glauben, Wissen und Wahrscheinlichkeit, Wien (Springer) 1980.

\section{Philosophische Fakultät I,}

Universität Regensburg, D-93040 Regensburg,

Germany. 